\title{
On LQG Control Across a Stochastic Packet-Dropping Link
}

\author{
Vijay Gupta, Demetri Spanos, Babak Hassibi and Richard M Murray
}

\begin{abstract}
In this paper, we consider the problem of optimal Linear Quadratic Gaussian control of a system in which communication between the sensor and the controller occurs across a packet-dropping link. We first prove a separation principle that allows us to solve this problem using a standard LQR state-feedback design, along with an optimal algorithm for propagating and using the information across the unreliable link. Then we present one such optimal algorithm, which consists of a Kalman filter at the sensor side of the link, and a switched linear filter at the controller side. Our design does not assume any statistical model of the packet drop events, and is thus optimal for any arbitrary packet drop pattern. Further, the solution is appealing from a practical point of view because it can be implemented as a small modification of an existing LQG control design.
\end{abstract}

\section{INTRODUCTION}

Recently, much attention has been directed toward systems which are controlled over a communication link (see, e.g., [1] and the references therein). In such systems, the control performance can be severely affected by the properties of the network or the channel. Communication links introduce many potentially detrimental phenomena, such as quantization error, random delays, data corruption and packet drops to name a few. In extreme cases, poor network performance can even destabilize a nominally stable control loop. Understanding and counter-acting these effects will become increasingly important as emerging applications of decentralized control mature. These applications will require the exchange of critical pieces of information over unreliable communication media.

The above issues have motivated much of the study of networked systems. Beginning with the seminal paper of Delchamps [5], quantization effects have been studied by Tatikonda [28], Elia and Mitter [6], Brockett and Liberzon [4], Hespanha et al. [12], Ishii and Francis [14], Nair and Evans [23], and many others. The effects of delayed packet delivery have also been considered in many works, such as Nilsson [24], Blair and Sworder [3], Luck and Ray [22], Tsai and Ray [29], and Zhang et al. [31], using various models for the network delay.

Division of Engineering and Applied Science, California Institute of Technology, Pasadena, CA 91125, USA \{gupta, demetri, hassibi, murray\}@ealtech.edu.

Work supported in part by AFOSR grant F49620-01-1-0460 and in part by NSF grant CCR-0326554 for the first author.

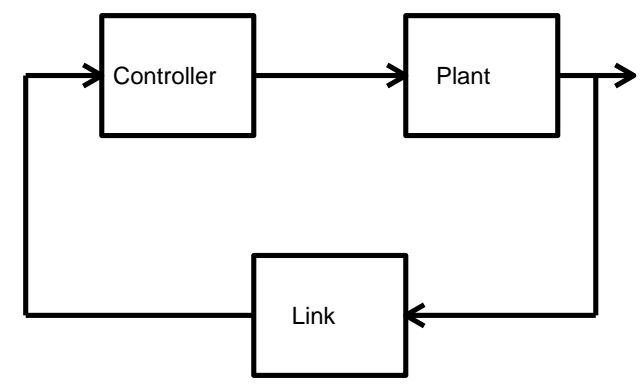

Fig. 1. The architecture of a packet-based control loop. The link is unreliable and unpredictably drops packets.

In this work, we are specifically interested in systems communicating over links that randomly drop packets. The nominal system is shown in Figure 1 where the link randomly drops packets being communicated from the plant to the controller. Preliminary work in this area studied stability of systems utilizing lossy packet-based communication, as in [10], [25], [31]. Performance of such systems as a function of packet loss rate was analyzed by Seiler in [25] and by Ling and Lemmon in [18] assuming certain statistical dropout models. Nilsson [24] proposed two approaches for compensation for data loss in the link by the controller, namely keeping the old control or generating a new control by estimating the lost data, and presented an analysis of the stability and performance of these approaches. Hadjicostis and Touri [9] analyzed the performance when lost data is replaced by zeros. Ling and Lemmon, in a series of papers [18], [19], [20], [17] proposed compensators for specific statistical data loss models. In [19], [17] they posed the problem of optimal compensator design for the case when data loss is independent and identically distributed (i.i.d.) as a nonlinear optimization. Azimi-Sadjadi [2] took an alternative approach and proposed a sub-optimal estimator and regulator to minimize a quadratic cost. Sinopoli et al. [27] and Imer et al. [13] extended this approach further to obtain optimal controllers when the packet drops were i.i.d. The related problem of optimal estimation across a packet-dropping link was considered by Sinopoli et. al in [26] and extended by Liu and Goldsmith in [21].

Most of the designs proposed in these references aim 


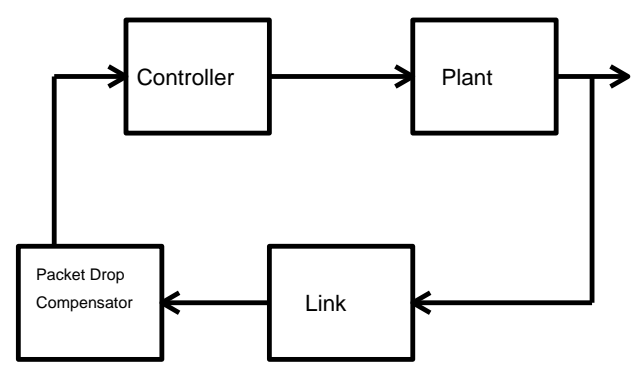

Fig. 2. A common design for control over packet-based links. The compensator aims at mitigating the effects of packet losses.

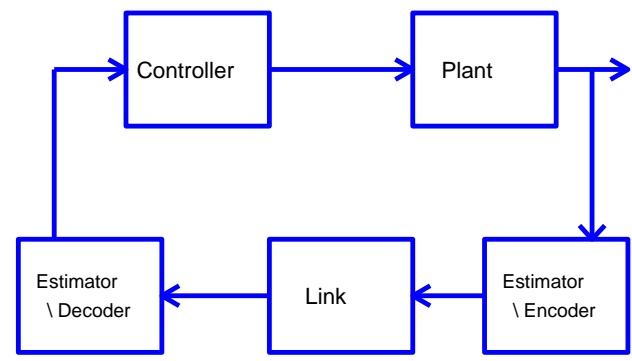

Fig. 3. The structure of our optimal LQG control solution.

at designing a packet-loss compensator, as shown in Figure 2. The compensator accepts those packets that the link successfully transmits and comes up with an estimate for the time steps when data is lost. This estimate is then used by the controller. Our work takes a more general approach by seeking the LQG optimal control for this packet-based problem. In particular, our architecture is as shown in Figure 3. We aim to jointly design the controller, the encoder and the decoder to solve the optimal LQG problem.

The remainder of this paper is organized as follows. In the next section, we present our mathematical model and pose the LQG problem in a packet-based setting. We then discuss a separation between control and estimation costs, and present an optimal solution to the estimation problem. We analyze the stability of our system and compare its performance with some other approaches in the literature. We finish by pointing out some directions for future research.

\section{PRoblem Formulation}

Consider a discrete-time linear system evolving according to

$$
x_{k+1}=A x_{k}+B u_{k}+w_{k},
$$

where $x_{k} \in \mathbf{R}^{n}$ is the process state, $u_{k} \in \mathbf{R}^{m}$ is the control input and $w_{k}$ is random noise entering the system. The noise process is assumed white, Gaussian, and zero mean with covariance matrix $Q_{w} .{ }^{1}$ The initial condition $x_{0}$ is assumed to be independent of $w_{k}$ and to have mean zero and covariance matrix $Q_{0}$. The state of the plant is measured by a sensor according to the equation

$$
y_{k}=C x_{k}+v_{k} .
$$

Here $v_{k}$ is the measurement noise, again assumed white, zero-mean, Gaussian (with covariance matrix $Q_{v}$ ) and independent of the plant noise $w_{k}$. The sensor communicates these measurements (or some function of the measurements) to the controller. We impose the constraint that the function communicated should be a finite vector, whose size does not increase with time. The communication is done over a channel that randomly drops packets. For the moment we ignore delays and packet reordering; it will be shown that these effects can be accounted for with time-stamping and a slight modification to our design. Thus, at each time-step $k$,

- A packet containing some function of the measurements is created at the sensor side of the link. We do not specify in advance what data these packets will contain.

- The packet is sent across the link.

- The packet is either received instantaneously, or dropped, probabilistically.

The packet dropping is a random process. We refer to individual (i.e. deterministic) realizations of this random process as packet drop sequences. A packet drop sequence is a binary sequence $\left\{\lambda_{k}\right\}_{k=0}^{\infty}$ in which $\lambda_{k}$ takes the value "received" if the link delivers the packet at time step $k$, and "dropped" if the packet is dropped.

We assume sufficient bits per packet and a high enough data rate so that quantization error is negligible. We also assume that enough error-correction coding is done within the packets so that the packets are either dropped or received without error. Finally, we assume no coding is done across packets; that is, no packet contains information about any other packet. We impose this constraint because Coding across packets can induce a large encoding and decoding delay which is undesirable for control applications.

The packetized communication link described above warrants some discussion regarding the class of controllers we will allow. The absolutely optimal LQG performance achievable is obviously given by the classical LQR controller/Kalman estimator pair. However, this design does not respect the packetized nature of the communication. Specifically, the controller requires continual access to the Kalman filter output, which in turn requires continual access to the sensor measurements.

\footnotetext{
${ }^{1}$ The results continue to hold for time-varying systems, but we consider the time-invariant case to simplify the presentation.
} 
This access might not be always possible because of data loss in the communication link. In order to make the class of controllers that are allowed more precise, we introduce the following terminology. Denote by $s_{k}$ the finite vector transmitted from the sensor to the controller at time step $k$. By causality, $s_{k}$ can depend (possible in a time-varying manner) on $y_{0}, y_{1}, \cdots, y_{k}$, i.e., $s_{k}=f_{k}\left(y_{0}, y_{1}, \cdots, y_{k}\right)$. The information set available to the controller at time $k$ is

$$
I_{k}=\left\{s_{k} \mid \forall k \text { s.t. } \lambda_{k}=\text { received }\right\}
$$

Also denote by $t_{l}(k) \leq k$ the last time-step at which a packet was delivered. The maximal information set at time-step $k$ is defined as follows:

$$
I_{k}^{\max }=\left\{y_{i} \mid 0 \leq i \leq t_{l}(k)\right\}
$$

The maximal information set is the largest set of output measurements on which the control at time-step $k$ can depend. In general, the set of output measurements on which the control depends will be less than this set, since earlier packets, and hence measurements, may have been dropped. The information contained in $I_{k}^{\max }$ upper bounds the information contained in $I_{k}$. We do not yet specify how the encoder designs or the controller uses $I_{k}$. As stated earlier, the only restriction we impose is that the vector $s_{k}$ not increase in size as $k$ increases. We will call the set of $f_{k}$ 's which fulfill this requirement as $\mathbf{F} .{ }^{2}$ Without loss of generality, we will only consider information-set feedback controllers, i.e. controllers of the form $u_{k}=u\left(I_{k}, k\right)$. Thus, we allow the control to depend on the information set, and on the current timeindex. Clearly, this is the broadest class of controllers one can sensibly consider for this problem since we have not assumed anything about the functional form of the control. Moreover, it is impossible for a physical realization of the controller to have more feedback information than is contained in the information set. We shall assume perfect knowledge of the system parameters $A, B, C$, $Q_{w}$ and $Q_{v}$ at the controller. Moreover we assume that the controller has access to the previous control signals $u_{0}, u_{1}, \cdots, u_{k-1}$ while calculating the control $u_{k}$ at time $k$. For notational convenience, we denote the set of control laws allowed by $U$.

\footnotetext{
${ }^{2}$ The information set is reminiscent of the 'information pattern' introduced by Witsenhausen [30]. We assume that the controller at time step $k$ has access to all the previous controls $u_{0}, u_{1}, \cdots, u_{k-1}$. Thus the 'information pattern' of the controller consists of the set $I_{k}$ and all the previous controls.
}

We can now pose the packetized LQG problem as follows:

$$
\begin{aligned}
& \min _{u \in U, f \in \mathbf{F}} J_{K}(u, f, P)=E\left[\sum_{k=0}^{K} u_{k}^{T} Q^{c} u_{k}\right] \\
& +E\left[\sum_{k=0}^{K} x_{k}^{T} R^{c} x_{k}\right]+E\left[x_{K+1}^{T} P_{K+1}^{c} x_{K+1}\right] .
\end{aligned}
$$

Here $K$ is the horizon on which the plant is operated and the expectation is taken over the uncorrelated variables $x_{0},\left\{w_{i}\right\}$ and $\left\{v_{i}\right\}$. Note that the cost functional $J$ above depends on the packet-drop sequence $P$. However, we do not average across packet-drop processes; the solution we will present is optimal for an arbitrary realization of the packet dropping process. That is, for any given packet-drop sequence $P$, the controller, encoder and decoder we propose will minimize $J(u, f, P)$ over the set of allowable controllers $U$ and allowable functions F. Because of this, we will occasionally suppress the packet-drop dependence in the cost functional, and merely write $J(u, f)$ or just $J$. We now present our solution to this problem.

Note that the usual addition and scalar multiplication operations make $U$ a vector space. For a given $f$ and $P$, $J(u, f, P)$ is a convex cost functional, and so existence of optima follows immediately. We make no claim regarding uniqueness of optimal control in this problem.

Our goal, then, is to solve the standard LQG problem with the additional complication of the packet-dropping link. While this may appear a small modification, it is unclear a priori, what the structure of the optimal control algorithm should be, and in what way the packetized link should be used through the design of the encoder and the decoder. We will show that one optimal algorithm is to utilize an LQR state-feedback design at the controller side, and to use the link to send the state estimates from a Kalman filter at the sensor side. The technical presentation closely mirrors the one given in [8].

\section{SePARATION OF CONTROL AND ESTIMATION}

In this section we briefly revisit the LQG separation principle in the packet-based setting. This will motivate the structure of our optimal controller/encoder design. Thus, consider the $K$-horizon cost functional given in (3). We need to choose $u_{0}, u_{1}, \cdots, u_{K}$ that minimize $J_{K}(u, f, P)$. Following [11], we gather terms that depend on the choice of $u_{K}$ and $x_{K}$ and write them 
as

$$
\begin{aligned}
& T_{K}=E\left[u_{K}^{T} Q^{c} u_{K}+x_{K}^{T} R^{c} x_{K}\right] \\
& +E\left[x_{K+1}^{T} P_{K+1}^{c} x_{K+1}\right] \\
& =E\left[\left[\begin{array}{ll}
u_{K}^{T} & x_{K}^{T}
\end{array}\right] \Delta\left[\begin{array}{l}
u_{K} \\
x_{K}
\end{array}\right]\right] \\
& +E\left[w_{K}^{T} P_{K+1}^{c} w_{K}\right] \\
& =S_{K}+O_{K}
\end{aligned}
$$

where

$$
\begin{aligned}
\Delta & =\left[\begin{array}{lr}
Q^{c}+B^{T} P_{K+1}^{c} B & B^{T} P_{K+1}^{c} A \\
A^{T} P_{K+1}^{c} B & R^{c}+A^{T} P_{K+1}^{c} A
\end{array}\right] \\
S_{K} & =E\left[\left[\begin{array}{ll}
u_{K}^{T} & x_{K}^{T}
\end{array}\right] \Delta\left[\begin{array}{l}
u_{K} \\
x_{K}
\end{array}\right]\right] \\
O_{K} & =E\left[w_{K}^{T} P_{K+1}^{c} w_{K}\right] .
\end{aligned}
$$

In the above equation, we have used the system dynamics given in (1) and the fact that the plant noise is zero mean. Thus we can write

$$
\begin{array}{r}
J_{K}(u, f, P)=E\left[\sum_{k=0}^{K-1} u_{k}^{T} Q^{c} u_{k}+\sum_{k=0}^{K-1} x_{k}^{T} R^{c} x_{k}\right] \\
+S_{K}+O_{K} .
\end{array}
$$

We aim to choose $u_{K}$ to minimize $J_{K}(u, f, P)$ for a given $f$. From (4), it is clear that the only term where the choice of $u_{K}$ can make a difference is $S_{K} . S_{K}$ can be written as

$$
\begin{aligned}
S_{K}=E\left[( u _ { K } - \overline { u } _ { K } ) ^ { T } R _ { e , K } ^ { c } \left(u_{K}\right.\right. & \left.\left.-\bar{u}_{K}\right)\right] \\
& +E\left[x_{K}^{T} P_{K}^{c} x_{K}\right]
\end{aligned}
$$

where

$$
\begin{gathered}
R_{e, K}^{c}=Q^{c}+B^{T} P_{K+1}^{c} B \\
P_{K}^{c}=R^{c}+A^{T} P_{K+1}^{c} A \\
-A^{T} P_{K+1}^{c} B\left(Q^{c}+B^{T} P_{K+1}^{c} B\right)^{-1} B^{T} P_{K+1}^{c} A
\end{gathered}
$$

and $\bar{u}_{K}$ is the standard optimal LQ control, $\bar{u}_{K}=$ - $\left(R_{e, K}^{c}\right)^{-1} B^{T} P_{K+1}^{c} A x_{K}$. In the absence of the packetized link, the controller could simply use the standard optimal control $\bar{u}_{K}$. However, as discussed before, this control law does not lie in the set of allowable solutions $U$ because it is not realizable for any non-trivial packetdropping sequence. Instead, we will calculate $u_{K}$ based on the information set $I_{K}$ (and the previous controls $u_{0}$, $u_{1}, \cdots, u_{K-1}$ that are assumed known to the controller) and choose it so as to minimize $S_{K}$. The control problem thus reduces to an optimal estimation problem. Given the information set at time $k, I_{k}$, we denote the linear least mean square (llms) estimate of a random variable
$\Gamma$ based on this information as $\hat{\Gamma}_{\mid I_{k}}{ }^{3}$ Then we can write the optimal control at time step $K$ as

$$
u_{K}=\hat{\bar{u}}_{K \mid I_{K}}=-\left(R_{e, K}^{c}\right)^{-1} B^{T} P_{K+1}^{c} A \hat{x}_{K \mid I_{K}} .
$$

Thus, we only need to find the llms estimate of $x_{K}$, given the information $I_{K}$ available to the controller. Note that since the information content in $I_{k}$ is upper bounded by the information contained in $I_{k}^{\max }$, the error in $\hat{x}_{K \mid I_{K}}$ is lower bounded by the error in calculating

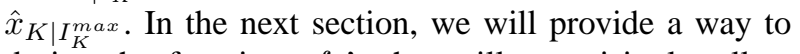
design the functions $f_{k}$ 's that will, surprisingly, allow the errors to actually coincide.

Denote the estimation error incurred due to the minimizing choice of $u_{K}$ by $\Lambda_{K}$. We have

$$
S_{K}=\Lambda_{K}+E\left[x_{K}^{T} P_{K}^{c} x_{K}\right] .
$$

Note that $\Lambda_{K}$ is independent of the previous control inputs $u_{0}, \cdots, u_{K-1}$ since these are assumed known to the controller when it calculates $u_{K}$ in (5). We can thus write the cost function as

$$
\begin{aligned}
J_{K}(u, f, P)= & E\left[\sum_{k=0}^{K-1} u_{k}^{T} Q^{c} u_{k}+\sum_{k=0}^{K-1} x_{k}^{T} R^{c} x_{k}\right] \\
& +S_{K}+O_{K} \\
= & E\left[\sum_{k=0}^{K-1} u_{k}^{T} Q^{c} u_{k}+\sum_{k=0}^{K-1} x_{k}^{T} R^{c} x_{k}\right] \\
& +\Lambda_{K}+E\left[x_{K}^{T} P_{K}^{c} x_{K}\right]+O_{K} \\
= & J_{K-1}(u, f, P)+\Lambda_{K}+O_{K} .
\end{aligned}
$$

Thus we now need to choose control inputs for time steps 0 to $K-1$ to minimize $J_{K-1}$, independently of the associated estimation cost at time step $K$ (the terms $O_{K}$ and $\Lambda_{K}$ do not involve these control inputs). However, our argument so far was independent of the time index $K$. Thus we can recursively apply this argument for time steps $K-1, K-2$ and so on. We have thus obtained the familiar separation result, in the packet-based setting:

Proposition 1 (Separation). Consider the packet-based optimal control problem defined in section II. For an optimizing choice of the control, the control and estimation costs decouple. Specifically, The optimal control input at time $k$ is calculated by using the relation

$$
u_{k}=\hat{\bar{u}}_{k \mid I_{k}}=-\left(R_{e, k}^{c}\right)^{-1} B^{T} P_{k+1}^{c} A \hat{x}_{k \mid I_{k}},
$$

where $\bar{u}_{k}$ is the optimal LQ control law while $\hat{\bar{u}}_{k \mid I_{k}}$ and $\hat{x}_{k \mid I_{k}}$ are the llms estimate of $\bar{u}_{k}$ and $x_{k}$ respectively,

\footnotetext{
${ }^{3}$ This notation is a bit misleading in that it suppresses the fact that the previous controls are known to the controller and are also used for the purpose of estimation. However we adopt it for simplicity and because it makes explicit the notion that the information quantity we aim to optimize over is contained in the set $I_{K}$. Also note that since all the random variables are Gaussian, and the cost function to be optimized is quadratic, the optimal estimator is linear.
} 
given the information set $I_{k}$ and the previous control laws $u_{0}, u_{1}, \cdots, u_{k-1}$.

This result must be viewed in light of the limited information available to the controller. At every time step, the controller tries to estimate the optimal control input based on the information set $I_{k}$, and uses this estimate in the optimal LQR control law. Thus, the state-feedback portion of an LQG controller need not be reworked for a packet-based implementation. The packet-based LQG question reduces to choosing what information should be sent from the sensor so that the optimal estimate can be formed at the controller, given that some of the packets might be lost. We address this issue in the next section.

\section{Optimal EnCODER AND Decoder Design}

In this section we present an algorithm for encoding and transmitting sensor measurements so as to achieve optimal estimation performance. Recall that we wish to construct the optimal estimate based on the information set $I_{k}^{\max }$, but we have not yet specified how to design $f_{k}$ 's that will allow the controller to compute that. For a link which does not drop packets, sending the current measurement $y_{k}$ in the current packet is sufficient. However, it is not clear that just sending the measurements can achieve optimality when packets are dropped. In particular, the Kalman filter input will be interrupted by the packet dropping. A naíve solution would be to send the entire history of the output variables at each time step. However, as mentioned earlier, this is not allowed since it requires increasing data transmission as time increases. Surprisingly, we can achieve performance equivalent to the naíve solution using a constant amount of transmission, and a constant amount of memory at the receiver end. We propose the following algorithm.

- The encoder (at the sensor end) receives as input the measurement $y_{i}$. It runs a Kalman filter that provides the llms estimate of $x_{k}$ based on all the measurements until time step $k$, denoted by $\hat{x}_{k \mid k}$ and transmits this vector across the link.

- The decoder (at the controller end) maintains a local variable $\hat{x}_{k}^{d e c}$. It is updated as follows:

- If $\lambda_{k}=$ received, the decoder receives $\hat{x}_{k \mid k}$, and sets $\hat{x}_{k}^{\text {dec }}=\hat{x}_{k \mid k}$.

- If $\lambda_{k}=$ dropped, then the decoder implements the following linear predictor:

$$
\hat{x}_{k}^{d e c}=A \hat{x}_{k-1}^{d e c}+B u_{k-1} .
$$

It is easy to see that if $\lambda_{k}=$ received, then $\hat{x}_{k}^{\text {dec }}$ is precisely the Kalman filter output, and thus is the optimal least-squares estimate of the state $x_{k}$. On the other hand if $\lambda_{k}=$ dropped, then (6) carries out the time update step of the Kalman filter and thus results in the optimal 1.1.m.s. estimate given the sensor measurements till time $t_{l}(k)$. Thus we can state the following.

Proposition 2 (Optimal Estimation). In the algorithm

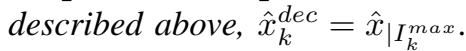

This, combined with our Proposition 1, allows us to state our main result.

Proposition 3 (Optimal Packet-Based LQG Control). For the packet-based optimal control problem stated in section II, an LQR state feedback design together with the optimal transmission-estimation algorithm described above achieves the minimum of $J(u, f, P)$ for any $P$.

Thus we have solved the packet-based LQG control problem posed in Section II. Note that we have made no assumption about the packet dropping behavior. The algorithm described above provides the optimal estimate based on $I_{k}^{\max }$ for an arbitrary packet drop sequence, irrespective of whether the packet drop can be modeled as an i.i.d. process (or a more sophisticated model like a Markov chain) or whether its statistics are known to the plant and the controller. Also note that the solution can be extended to the case when the channel applies a random delay to the packet so that packets might arrive at the decoder delayed or even out-of-order, if we assume that there is a provision for time-stamping the packets sent by the encoder. At each time step, the decoder will face one of four possibilities, and will update its estimate as described below:

- It receives $\hat{x}_{k \mid k}$. It uses this as its estimate.

- It does not receive anything. It uses the predictor equation (6) on $\hat{x}_{k-1}^{\text {dec }}$ as before.

- It receives $\hat{x}_{m \mid m}$ while at a previous time step, it has already received $\hat{x}_{n \mid n}$, where $n>m$. It discards $\hat{x}_{m \mid m}$ and uses the predictor equation (6) on $\hat{x}_{k-1}^{d e c}$.

- It receives $\hat{x}_{m \mid m}$ and at no previous time step has it received $\hat{x}_{n \mid n}$, where $n>m$. It uses $\hat{x}_{m \mid m}$ as $\hat{x}_{m}^{d e c}$ and uses the predictor equation until time step $k$ to obtain $\hat{x}_{k}^{\text {dec }}$.

\section{Analysis of the Proposed Algorithm}

In this section, we make some assumptions about the packet dropping random process and provide stronger results on the stability and performance of our algorithm. We model the channel erasures as occurring according to a Markov chain, which includes the case of independent packet drops as a special case. The model of Markov jump system is a popular way to deal with communication channels and networks. Thus the channel exists in either of two states, state 1 corresponding to a packet drop and state 2 corresponding to no packet drop and it transitions probabilistically between these states according to the transition probability matrix $Q$. We 
also assume strict causality in the Kalman filter used by the encoder. Thus to calculate the estimate of $x_{k}$, only the measurements till time step $k-1$ are used. The analysis can be easily extended to the case when strict causality is replaced by causality. Finally we assume that $(A, B)$ is stabilizable and the pair $(A, C)$ is detectable. We will denote the Kronecker product of matrices $A$ and $B$ by $A \otimes B$, while vec $(A)$ will represent the vectorizing operation that results in a vector formed by stacking the columns of $A$ (see [16] for more details).

\section{A. Stability Analysis}

The plant evolves as in (1), the Kalman filter at the encoder according to

$$
\hat{x}_{k+1}=A \hat{x}_{k}+B u_{k}+K_{k}\left(y_{k}-C x_{k}\right)
$$

and the estimator at the decoder according to the relation

$$
\hat{x}_{k+1}^{\text {dec }}= \begin{cases}A \hat{x}_{k}^{\text {dec }}+B u_{k} & \text { channel in state } 1 \\ \hat{x}_{k+1} & \text { channel in state } 2 .\end{cases}
$$

Denote $e_{k}=x_{k}-\hat{x}_{k}$ and $t_{k}=\hat{x}_{k}-\hat{x}_{k}^{\text {dec }}$. Since $u_{k}=$ $F_{k} \hat{x}_{k}^{d e c}$, (1) can be rewritten as

$$
x_{k+1}=\left(A+B F_{k}\right) x_{k}+w_{k}-B F_{k}\left(t_{k}+e_{k}\right) .
$$

If $(A, B)$ is stabilizable, by construction $F_{k}$ is the optimum control law and hence it stabilizes the system as long as the disturbances $w_{k}, t_{k}$ and $e_{k}$ remain bounded. We assume the noise $w_{k}$ has bounded covariance matrix. Also $e_{k}$ has bounded covariance matrices by assumption of detectability of $(A, C)$. Finally for $t_{k}$, we see that it evolves according to

$$
t_{k+1}= \begin{cases}A t_{k}+K v_{k}-K C e_{k} & \text { channel in state } 1 \\ 0 & \text { channel in state } 2\end{cases}
$$

Again note that $v_{k}$ and $e_{k}$ have bounded covariance. For $t_{k}$ to be of bounded variance, the Markov jump system of (7) needs to be stable. Further note that since our controller and encoder/decoder design is optimal, if the closed loop is unstable with our design, it is not stabilizable by any other design. Following [24], we can write the stability condition as follows.

Proposition 4 (Stability Condition). Consider the packet-based control problem defined in Section II in which the packet erasure channel is modeled as a Markov chain with transition probability matrix $Q$. Let the matrix pair $(A, B)$ be stabilizable and the matrix pair $(A, C)$ be detectable. The system is stabilizable, in the sense that the variance of the state is bounded, if and only if the matrix

$$
\left(Q^{T} \otimes I\right)\left[\begin{array}{lr}
0 & 0 \\
0 & A \otimes A
\end{array}\right]
$$

has eigenvalues strictly less than unity in magnitude, where $I$ is identity matrix and 0 is the zero matrix of suitable dimensions. Further, if the system is stabilizable, one controller and encoder/decoder design that stabilizes the system is given in Proposition 3.

As a simple example, suppose the channel has two states between which it jumps independently. With a probability $p$ at each time step, the channel drops the packet. Also assume that the plant is scalar with the system matrix given by $a$. Then the above condition reduces to the condition $p a^{2}<1$.

\section{B. Performance Analysis}

We now calculate the total quadratic cost incurred by the system for the infinite-horizon case (the case when $K \rightarrow \infty$ in (3)). We will make the additional assumption that the Markov chain is stationary and regular (see [7]) and that the stationary probability of channel being in state $i$ is given by $\pi(i)$. For the infinite horizon case, we consider the cost

$$
\begin{aligned}
J_{\infty} & =\lim _{K \rightarrow \infty} E\left[x_{K}^{T} R^{c} x_{K}+u_{K}^{T} Q^{c} u_{K}\right] \\
& =\operatorname{trace}\left(P_{x}^{\infty} R^{c}\right)+\operatorname{trace}\left(P_{u}^{\infty} Q^{c}\right),
\end{aligned}
$$

where $P_{x}^{\infty}=\lim _{K \rightarrow \infty} E\left[x_{K} x_{K}^{T}\right]$ and $P_{u}^{\infty}=$ $\lim _{K \rightarrow \infty} E\left[u_{K} u_{K}^{T}\right]$. With the assumptions of stability and detectability, the control law matrix $F_{k}$ and the Kalman gain matrix $K_{k}$ can be considered as constant matrices $F$ and $K$ respectively. From the discussion given in section $\mathrm{V}$-A, we can write the evolution of the system in the following manner. Denote $z_{k}=$ $\left[\begin{array}{ccc}x_{k}^{T} & e_{k}^{T} & t_{k}^{T}\end{array}\right]^{T}$ and $e_{k}=\left[\begin{array}{cc}w_{k}^{T} & v_{k}^{T}\end{array}\right]^{T}$. Then,

$$
z_{k+1}= \begin{cases}\mathbf{A}_{1} z_{k}+\mathbf{B}_{1} e_{k} & \text { channel in state 1 } \\ \mathbf{A}_{2} z_{k}+\mathbf{B}_{2} e_{k} & \text { channel in state 1, }\end{cases}
$$

where

$$
\begin{aligned}
& \mathbf{A}_{1}= {\left[\begin{array}{ccr}
A+B F & -B F & -B F \\
A-K C & 0 & 0 \\
0 & -K C & A
\end{array}\right] } \\
& \mathbf{A}_{2}= {\left[\begin{array}{ccr}
A+B F & -B F & -B F \\
A-K C & 0 & 0 \\
0 & 0 & 0
\end{array}\right] } \\
& \mathbf{B}_{1}=\left[\begin{array}{rr}
I & 0 \\
I & -K \\
0 & -K
\end{array}\right] \\
& \mathbf{B}_{2}=\left[\begin{array}{rr}
I & 0 \\
I & -K \\
0 & 0
\end{array}\right]
\end{aligned}
$$

while 0 denotes the zero matrix and $I$ the identity matrix of suitable dimensions. Define the stationary covariance $P^{\infty}=\lim _{k \rightarrow \infty} E\left[z_{k} z_{k}^{T}\right]$. Also denote $A_{1}=\mathbf{A}_{1} \otimes \mathbf{A}_{1}$, 
$A_{2}=\mathbf{A}_{2} \otimes \mathbf{A}_{2}, G_{1}=\mathbf{B}_{1} R \mathbf{B}_{1}^{T}, G_{2}=\mathbf{B}_{2} R \mathbf{B}_{2}^{T}, R=$ $E\left[e_{k} e_{k}^{T}\right]$ and $G=\left[\operatorname{vec}\left(G_{1}\right)^{T} \operatorname{vec}\left(G_{2}\right)^{T}\right]^{T}$. Finally define the conditional state covariance as

$$
\tilde{P}_{i}=\pi_{i} \lim _{k \rightarrow \infty} E\left[z_{k} z_{k}^{T} \mid \text { channel in state } i\right]
$$

so that $P^{\infty}=\tilde{P}_{1}+\tilde{P}_{2}$. Then we can use the results of [24] to obtain the following result.

Proposition 5 (Performance Analysis). Define $\tilde{P}=$ $\left[\operatorname{vec}\left(\tilde{P}_{1}\right)^{T} \operatorname{vec}\left(\tilde{P}_{2}\right)^{T}\right]^{T}$. Then $\tilde{P}$ is the unique solution to the linear equation

$$
\begin{aligned}
\tilde{P}=\left(Q^{T} \otimes I\right) & {\left[\begin{array}{cr}
A_{1} & 0 \\
0 & A_{2}
\end{array}\right] \tilde{P} } \\
& +\left(Q^{T} \otimes I\right)\left(\left[\begin{array}{rr}
\pi_{1} & 0 \\
0 & \pi_{2}
\end{array}\right] \otimes I\right) G,
\end{aligned}
$$

where $I$ is the identity matrix, 0 is the zero matrix and other quantities have been defined above.

Once we calculate $\tilde{P}$, we can readily evaluate the cost in (8) by using the relations

$$
\begin{aligned}
P_{x}^{\infty} & =\left[\begin{array}{lll}
I & 0 & 0
\end{array}\right] P^{\infty}\left[\begin{array}{l}
I \\
0 \\
0
\end{array}\right] \\
P_{u}^{\infty} & =F\left[\begin{array}{lll}
I & -I & -I
\end{array}\right] P^{\infty}\left[\begin{array}{c}
I \\
-I \\
-I
\end{array}\right] F^{T} .
\end{aligned}
$$

\section{Example}

In this section, we consider an example to illustrate the performance of our algorithm. We consider the example system considered by Ling and Lemmon in [17]. The plant transfer function is

$$
H(z)=\frac{z^{-1}+0.8 z^{-2}}{1+z^{-1}+1.7 z^{-2}},
$$

so that the system evolves as

$$
\begin{aligned}
x_{k+1} & =\left[\begin{array}{rr}
0 & -1.7 \\
1 & -1
\end{array}\right] x_{k}+\left[\begin{array}{c}
0.8 \\
1
\end{array}\right] u_{k}+\left[\begin{array}{l}
2 \\
1
\end{array}\right] w_{k} \\
y_{k} & =\left[\begin{array}{ll}
0 & 1
\end{array}\right] x_{k} .
\end{aligned}
$$

The process noise $w_{k}$ is zero mean with unit variance and the packet drop process is i.i.d. The cost considered is the steady state output error $\lim _{K \rightarrow \infty} y_{K}^{2}$. [17] assumes unity feedback when packets are delivered and gives an optimal compensator design when packets are being lost.

On analyzing the system with our algorithm, we observe that our algorithm allows the system to be stable up to a packet drop probability of 0.59 while the optimal compensator in [17] is stable only if the probability is less than 0.3 . Figure 4 shows the stability margin of our algorithm as a function of the packet drop probability.

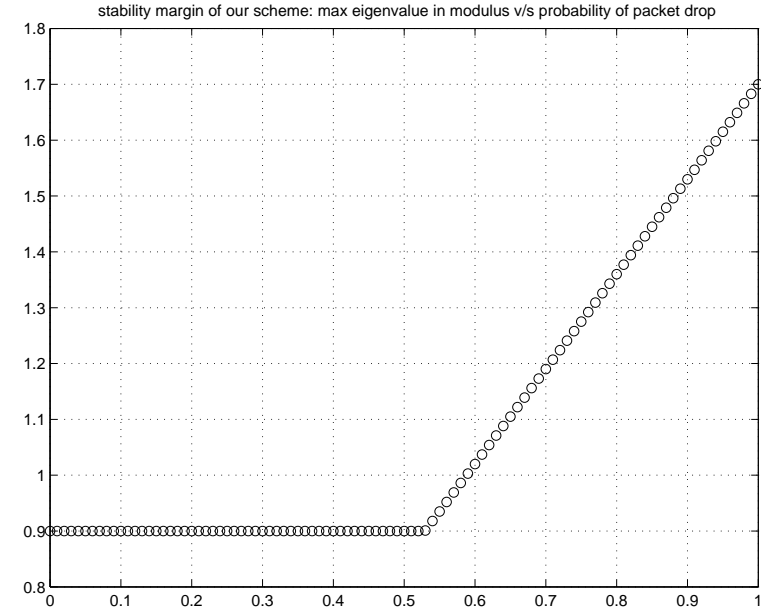

Fig. 4. Stability margin of our algorithm as a function of packet drop probability.

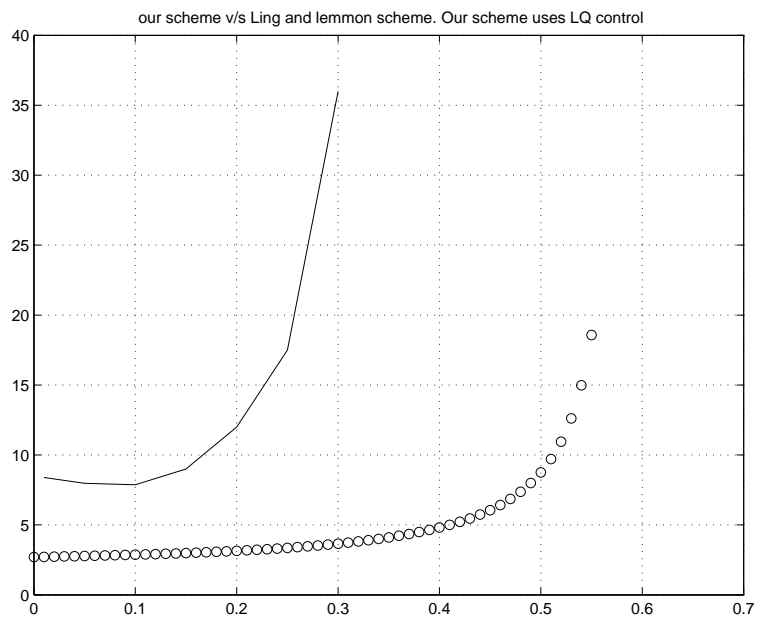

Fig. 5. Comparison of performance for the two algorithms assuming optimal controller for our algorithm.

It shows the maximum absolute value of the eigenvalue of the matrix given in Proposition 4. Also if we analyze the performance we obtain the plot given in Figure 5. The performance is much better throughout the range of operation for our algorithm. The performance of the two algorithms is not the same even at zero probability of packet drop since the optimal compensator presented in [17] assumes unity feedback when no packets are being dropped. Thus we see that the algorithm proposed in this paper performs better throughout the range of proportion than similar approaches proposed in the literature.

\section{CONClusions AND Future WORK}

In this paper, we considered the problem of optimal LQG control when the sensor and controller are commu- 
nicating across a channel or a network. We modeled the link as a switch that drops packets randomly and proved that a separation exists between the optimal estimate and the optimal control law. For the optimal estimate, we identified the information that the sensor should provide to the controller. This can be viewed as constructing an encoder for the channel. We also designed the decoder that uses the information it receives across the link to construct an estimate of the state of the plant. The proposed algorithm is optimal irrespective of the packet drop pattern. For the case of packet drops occurring according to a Markov chain, we carried out stability and performance analysis of our algorithm.

The work can potentially be extended in many ways. One possible direction is to consider a channel between the controller and the actuator. Another intriguing possibility is considering the effect of allowing only finite number of bits in the packet. Ishwar et al. [15] have showed that if the optimal vector to send in the infinite rate case is the state estimate, even for the finite rate case, the quantized version of state estimate remains as the optimal thing to send. However, from the view of optimal control, this issue has to be examined in greater detail. Extensions to decentralized control are another exciting avenue of research.

\section{REFERENCES}

[1] Special issue on networks and control. IEEE Control Systems Magazine, 21(1), Feb 2001.

[2] B. Azimi-Sadjadi. Stability of networked control systems in the presence of packet losses. In Proceedings of 2003 IEEE Conference on Decision and Control, Dec 2003.

[3] W.P. Blair and D.D. Sworder. Feedback control of a class of linear discrete systems with jump parameters and quadratic cost criteria. Int. J. Contr., 21(5):833-841, 1975.

[4] R. W. Brockett and D. Liberzon. Quantized feedback stabilization of linear systems. IEEE Transactions on Automatic Control, 45(7):1279-89, 2000.

[5] D. F. Delchamps. Stabilizing a linear system with quantized state feedback. IEEE Transactions on Automatic Control, 35:916-924, 1990.

[6] N. Elia and S. K. Mitter. Stabilization of linear systems with limited information. IEEE Transactions on Automatic Control, 46(9):1384-1400, 2001.

[7] J. E. Elliot, L. Aggoun, and J. B. Moore. Hidden Markov Models: Estimation and Control. Springer-Verlag, 1995.

[8] V. Gupta, D. Spanos, B. Hassibi, and R. M. Murray. Optimal LQG control across a packet-dropping link. IEEE Transactions on Automatic Control, 2004. Submitted.

[9] C. N. Hadjicostis and R. Touri. Feedback control utilizing packet dropping network links. In Proc. of the IEEE Conference on Decision and Control, 2002.

[10] A. Hassibi, S. P. Boyd, and J. P. How. Control of asynchronous dynamical systems with rate constraints on events. In Proc. IEEE Conf. Decision and Control, pages 1345-1351, Dec 1999.

[11] B. Hassibi, A. H. Sayed, and T. Kailath. Indefi nite-Quadratic Estimation and Control. Studies in Applied and Numerical Mathematics, 1999.

[12] J. Hespanha, A. Ortega, and L. Vasudevan. Towards the control of linear systems with minimum bit-rate. In Proc. of the 15th Int. Symp. Math. The. Netw. Sys., 2002.
[13] O. C. Imer, S. Yuksel, and T. Basar. Optimal control of dynamical systems over unreliable communication links. In NOLCOS 2004 Stuttgart,Germany, 2004.

[14] H. Ishii and B. A. Francis. Quadratic stabilization of sampleddata systems with quantization. Automatica, 39:1793-1800, 2003.

[15] P. Ishwar, R. Puri, K. Ramchandran, and S. S. Pradhan. On rateconstrained distributed estimation in unreliable sensor networks. submitted to the IEEE Journal on Selected Areas in Communications: Special issue on self-organizing distributed collaborative sensor networks, Dec 2003.

[16] P. Lancaster. Theory of Matrices. Academic Press, 1969.

[17] Q. Ling and M. D. Lemmon. Power spectral analysis of netwroked control systems with data droputs. IEEE Transactions on Automatic control, 49(6):955-960, June 2004.

[18] Q. Ling and M.D. Lemmon. Robust performance of soft realtime networked control systems with data dropouts. In Proc. of the IEEE Conference on Decision and Control, 2002.

[19] Q. Ling and M.D. Lemmon. Optimal dropout compensation in networked control systems. In Proc. of the IEEE Conference on Decision and Control, 2003

[20] Q. Ling and M.D. Lemmon. Soft real-time scheduling of networked systems with dropouts governed by a Markov chain. In Proc. of the American Conference on Control, 2003.

[21] X. Liu and A. J. Goldsmith. Kalman filtering with partial observation losses. IEEE Transactions on Automatic Control. submitted.

[22] R. Luck and A. Ray. An observer-based compensator for distributed delays. Automatica, 26(5):903-908, 1990

[23] G. N. Nair and R. J. Evans. Stabilizability of stochastic linear systems with finite feedback data rates. SIAM Journal on Control and Optimization, 2004. accepted.

[24] J. Nilsson. Real-Time Control Systems with Delays. PhD thesis, Department of Automatic Control, Lund Institute of Technology, 1998.

[25] P. Seiler. Coordinated Control of unmanned aerial vehicles. $\mathrm{PhD}$ thesis, University of California, Berkeley, 2001.

[26] B. Sinopoli, L. Schenato, M. Franceschetti, K. Poolla, M. Jordan, and S. Sastry. Kalman filtering with intermittent observations. IEEE Transactions on Automatic Control, Sep 2004.

[27] B. Sinopoli, L. Schenato, M. Franceschetti, K. Poolla, and S. S. Sastry. Time varying optimal control with packet losses. In IEEE Conference on Decision and Control, Bahamas, 2004. To be Presented.

[28] S. Tatikonda. Control under Communication Constraints. PhD thesis, MIT, Cambridge, MA, 2000.

[29] N. C. Tsai and A. Ray. Stochastic optimal control under randomly varying distributed delays. International Journal of Control, 68(5):1179-1202, Nov 1997.

[30] H. S. Witsenhausen. Separation of estimation and control for discrete time systems. Proceedings of the IEEE, 59(11):15571566, 1971.

[31] W. Zhang, M. S. Branicky, and S. M. Philips. Stability of networked control systems. IEEE Control System Magazine, 21(1):84-89, Feb 2001 